\title{
Extrapulmonary Causes of Acute Respiratory Failure-Early Diagnosis Can Drastically Improve Outcome
}

\author{
Dr Monila Patel*, Dr Itesh Khatwani**, Dr Bhagyadhan Patel** \\ *Associate Professor Of Department Of Medicine, VS Hospital-Ahmedabad, India \\ **3 $3^{\text {rd }}$ Year Resident Of Department Of Medicine, VS Hospital-Ahmedabad, India
}

\begin{abstract}
Extrapulmonary causes of respiratory failure include conditions that exclusively or primarily cause respiratory failure by their effects on structures other than the lungs i.e. the extra pulmonary compartment. They are mainly responsible for the hypercapnic respiratory failure. An analysis of 50 such patients was done at our I.C.U. We tried studying their clinical presentation, their causes and the outcome. The most common age group was 21-40 yrs with a male preponderance. Autonomic dysfunction, ophthalmoplegia, motor and bulbar weakness were the common presentations. Many of them had single breath count $(S B C)<20$ on presentation. Most common causes were neurotoxic snake bites and Organophosphorus poisoning. The outcome was relatively good if patients were brought early.
\end{abstract}

Keywords: hypercapnea, hypoxemia and type II respiratory failure

\section{Introduction}

Respiratory failure is a syndrome in which the respiratory system fails in one or both of its gas exchange functions: oxygenation and carbon dioxide elimination. In practice, it may be classified as either hypoxemic or hypercapnic

Hypercapnic respiratory failure (type II) is characterized by a $\mathrm{PaCO} 2$ (arterial carbon dioxide tension) higher than $50 \mathrm{~mm} \mathrm{Hg}$. Hypoxemia is common in patients with hypercapnic respiratory failure who are breathing room air. The $\mathrm{pH}$ depends on the level of bicarbonate, which, in turn, is dependent on the duration of hypercapnia. Common etiologies include neuroparalytic snake bite, drug overdose, neuromuscular disease, chest wall abnormalities and severe airway disorders (eg, asthma and chronic obstructive pulmonary disease [COPD]) [1].The causes of hypercapnic respiratory failure can be divided into two main groups: extrapulmonary and pulmonary. Extrapulmonary causes of respiratory failure include conditions that exclusively or primarily cause respiratory failure by their effect on structures other than the lungs (i.e. the extrapulmonary compartment) The following components make up extrapulmonary compartment : a ) central nervous system $b$ ) peripheral nervous system c ) respiratory muscles d) chest wall e ) pleura f) airway. Extrapulmonary causes of respiratory failure are important for several reasons. First, although precise figures are not available, it has been estimated that extrapulmonary causes can account for up to one sixth of all cases of hypercapnic respiratory failure. Second, extrapulmonary causes are often overlooked or misdiagnosed. The sine qua non of pure extrapulmonary respiratory failure is arterial hypercapnia and a normal A-aPO2 gradient (alveolar-arterial oxygen tension gradient) [2]. The normal gradient reflects the fact that in pure extrapulmonary failure distal gas exchange is entirely normal, and the decrease in $\mathrm{PaO} 2$ (arterial oxygen tension) directly reflects the decrease in the PAO2. An A-a P02 gradient less than $20 \mathrm{~mm} \mathrm{Hg}$ in the presence of an elevated $\mathrm{PaCO} 2$ is virtually diagnostic of extrapulmonary respiratory failure [3]

It is important to understand the common clinical presentations and the extrapulmonary causes of respiratory failure as most of them hold a good prognosis if brought early.

\section{Material and Methods}

This study was done at the department of medicine and department of emergency medicine, V.S. hospital Ahmedabad. Number of patients studied was 50. The patients selected were older than $12 \mathrm{yrs,} \mathrm{had} \mathrm{an}$ extrapulmonary cause of respiratory failure and had clinical signs or laboratory parameters $\left(\mathrm{P}_{\mathrm{co} 2}>50, \mathrm{P}_{\mathrm{o} 2}<60\right)$ suggestive of respiratory failure. Patients with a history of chronic respiratory disease, a past history of CVA or the ones who presented with ARDS were excluded from the study.

Detailed history of all patients was taken in form of age, sex, BMI, history of co morbid illness such as hypertension, diabetes mellitus, Ischemic heart disease, history of snake bite, time to hospitalization from time of bite, ingestion of substance. General examination in form of Heart rate, respiratory rate was taken. Complete neurological examination in form of consciousness (GCS-Glasgow Coma Scale), ophthalmoplegia, bulbar weakness, neck muscle and limb muscle weakness, fasciculations, reflexes, sensory modalities (joint position sense, pain, temperature ) and autonomic functions ( pupils, bowel bladder, postural hypotension and vasomotor symptoms ) was taken. Modified RANKIN score was applied on discharge. 
Modified RANKIN score

0 - No symptoms

1 - No significant disability. Able to carry out all usual activities, despite some symptoms.

2 - Slight disability. Able to look after own affairs without assistance, but unable to carry out all previous activities.

3 - Moderate disability. Requires constant nursing care and attention

4 - Moderately severe disability. Unable to attend to own bodily needs without assistance, and unable to walk unassisted.

5 - Severe disability. Requires constant nursing care and attention, bedridden, incontinent.

6 - Dead [4]

Clinical features of Respiratory Failure and the Need for Endotracheal Intubation [5]

1) General Warning Signs

a. Increasing generalized weakness

b. Dysphagia

c. Dysphonia

d. Dyspnea on exertion and at rest

2) Subjective Assessment

a. Rapid shallow breathing

b. Tachycardia

c. Weak cough

d. Staccato speech

e. Accessory muscle use

f. Abdominal paradox

g. Orthopnea

h. Weakness of trapezius and neck muscles

i. Single-breath count $(\mathrm{SBC})<20$

j. Cough after swallowing

Laboratory investigations, namely hemoglobin, total leucocyte count, platelet count, serum creatinine, serum glutamate pyruvate transaminase (SGPT), electrolytes like sodium, potassium, calcium, magnesium, phosphate, arterial blood gas analysis including $\mathrm{pH}, \mathrm{Paco} 2, \mathrm{Pao} 2$ were carried out in all patients. Other blood investigations carried out in selected patients were serum cholinesterase, antibody to acetylcholine receptor, anti aquaporin 4 antibodies, ANA, ANA BLOT, PT/aPTT, CPK total, TSH, fasting lipid levels.

Radiological investigations inform of Chest X-RAY was carried out in all patients. Few selected patients underwent USG abdomen and KUB, 2DEcho, MR Angio of brain, MRI cervical spine, CT brain plain and other miscellaneous investigations as required.

\section{Results}

The data was tabulated and analyzed using percentage and proportion

3.1. CLINICAL PROFILE OF PATIENTS WITH EXTRAPULMONARY CAUSES OF RESPIRATORY FAILURE (TABLE 1A and 1B)

The most common clinical sign was autonomic involvement in form of variation in pupil size was found in $58 \%$ of patients followed by limb weakness (50\%), bulbar weakness (48\%). Other clinical features were respiratory muscle weakness $(\mathrm{SBC}<20)$, ophthalmoplegia, neck flexor weakness and fasciculations. The pupillary size being most common clinical sign was attributed to OP poisoning and neuroparalytic snake bite which are most common extrapulmonary diseases causing respiratory failure.

In neuroparalytic snake bite most common clinical sign in our study was autonomic dysfunction( $82 \%)$ while in Seneviratne et al study [6] also it was autonomic dysfunction( $84 \%)$

\subsection{CLINICAL SIGNS OF RESPIRATORY FAILURE(TABLE 2)}

$\mathrm{SBC}<20$ was found in $42 \%$ of the patients followed by other signs suggesting its importance in early diagnosis.

\subsection{ETIOLOGY OF EXTRAPULMONARY CAUSES OF RESPIRATORY FAILURE IN STUDY PATIENTS (TABLE 3)}

The most common extrapulmonary disease causing respiratory failure in our study was neuroparalytic snake bite (34\%), followed by OP poisoning (20\%). While in study by Agarwal et al [7], it was neuroparalytic snake bite (36\%), followed by OP poisoning (14\%). Other causes were GBS, MG, tetanus and others. 


\subsection{ABGA ANALYSIS OF STUDY PATIENTS (TABLE 4)}

The most common respiratory failure was pure type 2 in $54 \%$ of the patients followed by mixed (type 1 plus type 2) in $32 \%$.

\subsection{DISTRIBUTION OF MODIFIED RANKIN SCORE IN STUDY PATIENTS(ON DISCHARGE)(TABLE 5)}

Complete recovery was found in neuroparalytic snake bite, OP poisoning and hypokalemic paralysis without any disability. Moderate disability was found in patients with GBS, MG and other neuromuscular disorders.

In our study mortality rate was highest for brainstem lesions (100\%), while in Agarwal et al [7] study it was also highest for brainstem lesions (50\%). Mortality was nil for MG in both the studies.

\section{Discussion}

Impairment of extrapulmonary compartment produces respiratory failure through the mechanism of hypoventilation; resultant respiratory failure is usually hypercapnic. Extrapulmonary causes can account for up to an estimated $17 \%$ of all cases of hypercapneic failure. Functionally,extrapulmonary disordes lead to hypercapneic respiratory failure because of a decrease in normal force generation (CNS dysfunction,peripheral nervous system abnormalities or respiratory muscle dysfunction) or an increase in impedance to bulk flow ventilation (chest wall and pleural disorders or upper airway obstruction).

Any condition that directly or indirectly impairs respiratory muscle function can result in decreased force generation. If this impairment is severe enough, the level of minute ventilation may be insufficient for the level of production of carbon dioxide [8]

A decrease in central drive to breathe may occur from direct central loss of sensitivity to changes in Paco2 and $\mathrm{pH}$ or as a result of a peripheral chemoreceptor loss of sensitivity to hypoxia, as with CNS depressants (narcotics, barbiturates), metabolic abnormalities (hypothyroidism, starvation, metabolic alkalosis), CNS structural lesions, primary alveolar hypoventilation and central sleep apnea.

Disruption of impulse transmission from the respiratory centre in the brainstem to the respiratory muscles may result in respiratory failure. The innervations of the inspiratory respiratory muscles may be involved as part of a generalized process, such as in Gullian Barre syndrome (GBS), myasthenia gravis, amyotrophic lateral sclerosis (ALS), neuromuscular junction blockade, or as an isolated abnormality that affects the respiratory system in a variable way depending on the level of the injury, such as in phrenic nerve palsy and spinal cord trauma or lesions.

Peripheral nervous system dysfunction severe enough to produce hypercapneic respiratory failure is always associated with a reduced vital capacity (usually $<50 \%$ of predicted value) and markedly decreased maximal inspiratory pressure(MIP) and maximal expiratory pressure (MEP) at the mouth (usually $<30 \%$ of predicted).A partial list of the myriad etiologies in this group include ALS, poliomyelitis, GBS, shellfish poisoning, diphtheria, tick paralysis, myasthenia gravis, Eaton-Lambert syndrome, critical illness polyneuropathy, botulism, snake bite, tetanus and O-P poisoning.

Certain systemic myopathies feature prominent respiratory muscle involvement, such as muscular dystrophies, myotonic disorders, inflammatory and endocrine myopathies, and electrolyte disturbances (hypophosphatemia, hyper/hypomagnesemia, hypokalemia6, hypercalcemia).

Any disorder that causes a decrease in chest wall or pleural compliance (kyphoscoliosis, pleural fibrosis, flail chest, obesity-hypoventilation syndrome, ankylosing spondylitis) or increases airflow resistance from upper airway obstruction (foreign body aspiration, tracheal stenosis, epiglottis, laryngeal edema, laryngeal/tracheal tumors, obstructive sleep apnea) may culminate in hypercapneic respiratory failure if the resultant respiratory muscle force requirements cannot be sustained [2]

\section{Conclusion}

The most common clinical feature was autonomic dysfunction in form of pupillary involvement (58\%) followed by limb weakness $(50 \%)$

The most common clinical sign of respiratory failure was $\mathrm{SBC}<20$, which stresses its importance in routine monitoring of these patients.

The extrapulmonary causes of respiratory failure in our study in descending order were NP snake bite (34\%), OP poisoning (20\%), GBS (12\%), and MG (8\%).

Complete recovery was found in NP snake bite (especially those presenting early), OP poisoning and hypokalemic paralysis without any disability.

Moderate disability was seen in GBS, MG and other NM disorders.

Mortality rates were high in brainstem lesions and critical illness neuropathy. 
TABLES

Table 1A: CLINICAL PROFILE OF PATIENTS WITH EXTRAPULMONARY CAUSES OF RESPIRATORY FAILURE

\begin{tabular}{|l|l|l|}
\hline CLINICAL PROFILE & NO. OF PATIENTS & $\%$ \\
\hline OPHTHALMOPLEGIA & 20 & 40 \\
\hline BULBAR WEAKNESS & 24 & 48 \\
\hline NECK FLEXOR WEAKNESS & 19 & 38 \\
\hline SBC $<20$ & 21 & 42 \\
\hline LIMB WEAKNESS & 25 & 50 \\
\hline FASCICULATIONS & 4 & 8 \\
\hline AUTONOMIC INVOLVEMENT & 29 & 58 \\
\hline
\end{tabular}

Table 1B:

\begin{tabular}{|l|l|l|}
\hline GCS SCORE & NO. OF PATIENTS & $\%$ \\
\hline GCS 3-4 & 2 & 4 \\
\hline GCS $5-11$ & 11 & 22 \\
\hline GCS $>11$ & 37 & 74 \\
\hline
\end{tabular}

Table 2: CLINICAL SIGNS OF RESPIRATORY FAILURE

\begin{tabular}{|l|l|l|}
\hline CLINICAL SIGNS & NO. OF PATIENTS & $\%$ \\
\hline SBC $<20$ & 21 & 42 \\
\hline RAPID SHALLOW BREATHING $>25$ & 13 & 26 \\
\hline USE OF ACCESSORY MUSCLES & 13 & 26 \\
\hline STACCATO SPEECH & 13 & 26 \\
\hline ABDOMINAL PARADOX & 13 & 26 \\
\hline ORTHOPNEA & 8 & 16 \\
\hline
\end{tabular}

Table 3: ETIOLOGY OF EXTRAPULMONARY CAUSES OF RESPIRATORY FAILURE IN STUDY PATIENTS

\begin{tabular}{|l|l|l|}
\hline ETIOLOGY & NO. OF PATIENTS & $\%$ \\
\hline NEUROPRALYTIC SNAKE BITE & 17 & 34 \\
\hline OP POISONING & 10 & 20 \\
\hline GBS & 6 & 12 \\
\hline MG & 4 & 8 \\
\hline HYPOKALEMIC PARALYSIS & 3 & 6 \\
\hline TETANUS & 3 & 6 \\
\hline BRAINSTEM LESIONS & 2 & 4 \\
\hline OTHER NEUROMUSCULAR DISORDERS & 2 & 4 \\
\hline CERVICAL MYELOPATHY & 1 & 2 \\
\hline OBSTRUCTIVE SLEEP APNOEA & 1 & 2 \\
\hline CHEST WALL DISORDERS & 1 & 2 \\
\hline
\end{tabular}

Table 4: ABGA ANALYSIS OF STUDY PATIENTS

\begin{tabular}{|l|l|l|}
\hline ABGA PARAMETERS & NO. OF PATIENTS & $\%$ \\
\hline $\mathrm{PO} 2<60 \mathrm{~mm} \mathrm{Hg}$ & 3 & 6 \\
\hline $\mathrm{PCO} 2>45 \mathrm{~mm} \mathrm{Hg}$ & 27 & 54 \\
\hline $\mathrm{PO} 2<60 \mathrm{~mm} \mathrm{Hg}$ and $\mathrm{PCO} 2>45 \mathrm{mmHg}$ & 16 & 32 \\
\hline $\mathrm{PO} 2>60 \mathrm{~mm} \mathrm{Hg}$ and $\mathrm{PCO} 2<45 \mathrm{mmHg}$ & 4 & 8 \\
\hline
\end{tabular}

Table 5: DISTRIBUTION OF MODIFIED RANKIN SCORE IN STUDY PATIENTS (ON DISCHARGE)

\begin{tabular}{|c|c|c|c|c|c|c|c|c|c|c|}
\hline & \multicolumn{2}{|l|}{ SCORE 0} & \multicolumn{2}{|l|}{ SCORE 1} & \multicolumn{2}{|l|}{ SCORE 2} & \multicolumn{2}{|l|}{ SCORE 3-5 } & \multicolumn{2}{|l|}{ SCORE 6} \\
\hline DISEASES & $\begin{array}{l}\text { NO. OF } \\
\text { PATIENTS }\end{array}$ & $\%$ & $\begin{array}{l}\text { NO. OF } \\
\text { PATIENTS }\end{array}$ & $\%$ & $\begin{array}{l}\text { NO. OF } \\
\text { PATIENTS }\end{array}$ & $\%$ & $\begin{array}{l}\text { NO. OF } \\
\text { PATIENTS }\end{array}$ & $\%$ & $\begin{array}{l}\text { NO. OF } \\
\text { PATIENTS }\end{array}$ & $\%$ \\
\hline $\begin{array}{l}\text { NEUROPARALYTIC } \\
\text { SNAKE BITE }\end{array}$ & 16 & 94.2 & 0 & 0 & 0 & 0 & 0 & 0 & 1 & 5.8 \\
\hline OP POISONING & 8 & 80 & 1 & 10 & 0 & 0 & 0 & 0 & 1 & 10 \\
\hline GBS & 0 & 0 & 2 & 33.3 & 1 & 16.7 & 2 & 33.3 & 1 & 16.7 \\
\hline MG & 0 & 0 & 2 & 50 & 1 & 16.7 & 1 & 16.7 & 0 & 0 \\
\hline $\begin{array}{l}\text { BRAINSTEM } \\
\text { LESIONS }\end{array}$ & 0 & 0 & 0 & 0 & 0 & 0 & 0 & 0 & 2 & 100 \\
\hline CIP & 0 & 0 & 0 & 0 & 0 & 0 & 0 & 0 & 1 & 100 \\
\hline $\begin{array}{l}\text { OTHER } \\
\text { NEUROMUSCULAR } \\
\text { DISEASES }\end{array}$ & 0 & 0 & 0 & 0 & 0 & 0 & 1 & 50 & 0 & 0 \\
\hline $\begin{array}{l}\text { CERVICAL } \\
\text { MYELOPATHY }\end{array}$ & 0 & 0 & 0 & 0 & 0 & 0 & 1 & 100 & 0 & 0 \\
\hline $\begin{array}{l}\text { HYPOKALEMIC } \\
\text { PARALYSIS }\end{array}$ & 3 & 100 & 0 & 0 & 0 & 0 & 0 & 0 & 0 & 0 \\
\hline
\end{tabular}


Extrapulmonary Causes Of Acute Respiratory Failure-Early Diagnosis Can Drastically Improve

\begin{tabular}{|l|l|l|l|l|l|l|l|l|l|l|}
\hline TETANUS & 0 & 0 & 1 & 33.3 & 2 & 66.7 & 0 & 0 & 0 & 0 \\
\hline OSA & 0 & 0 & 0 & 0 & 1 & 100 & 0 & 0 & 0 & 0 \\
\hline $\begin{array}{l}\text { CHEST WISORDERS } \\
\text { TOTAL }\end{array}$ & 0 & 0 & 1 & 100 & 0 & 0 & 0 & 0 & 0 & 0 \\
\hline
\end{tabular}

\section{References}

[1] Respiratory failure.(2012).at http://emedicine.medscape.com/article/167981-overview

[2] Demers RR, Irwin RS328-353(1979)

[3] Pratter, M.R. and Irwin, R.S. Extrapulmonary causes of respiratory failure. J intensive care med 1,197-207(1986)

[4] CMC .Modified Rankin scale.doc.at <http:/www.encsn.org/hospitals plans of care templates/CMC.modifiedrankinscale.doc $>$

[5] Neuromuscular disease causing respiratory failure.at $\langle$ http://rc.journal.com/content/51/9/1016.abstract $>$

[6] Pail ,V., Pail, H.,A and Agrawal, V. Clinical profile and outcome of envenomous snake bite at tertiary care centre. International journal of medicine and public health 1 28-38(2011)

[7] Aggarwal, A.N. non pulmonary organ dysfunction and its impact on outcome in patients with acute respiratory failure $<x r e f$ rid ='AFF1'></xref $>$.chest journal 132,829(2007)

[8] T.N.S, Welte respiratory emergencies. (European respiratory society, 2006) 